\title{
Characterization of fatigue resistance property of photochromic materials for optical storage devices
}

\author{
Elena Samoylova ${ }^{1 *}$, Member, IEEE, Marco Allione ${ }^{1}$, Alberto Diaspro $^{1}$, Member, IEEE, Roberto Cingolani ${ }^{1}$, Athanassia \\ Athanassiou ${ }^{2}$
}

\begin{abstract}
Diarylethenes were proposed as very promising candidates for optical memory applications due to their thermal stability and fatigue resistance. The latter property of two photochromic molecules of the diarylethene family embedded in polymer films was investigated in this work. The obtained results demonstrate a noticeable influence of the polymer matrix and possible photochemical damage on the fatigue resistance of the photochromic materials.
\end{abstract}

\section{BACKGROUND}

Photochromic molecules have received a lot of attention in recent years due to their potential application for optical devices such as $3 \mathrm{D}$ optical memories and switches. Wide range of photochromic molecules have been investigated and described in the literature, such as fulgides [1-3], fulgimides [4], spiropyrans [6-9], diarylethenes [10-12]. Implementation of these molecules into polymeric matrices with different properties allows to fabricate small, cheap and sensitive photochromic devices. Photochromic materials should fulfill several practical requirements, among which are thermostability and good resistance to photochemical fatigue (can be recycled many time without significant loss of performance). Among the most promising photochromic molecules presented in the literature are diarylethene derivatives due to their excellent thermo- and photostability [9] As shown by Irie theoretically and experimentally [9], both open and closed forms of compounds $\mathbf{1}$ and $\mathbf{2}$ are thermally stable (more than 12 hours at $80^{\circ} \mathrm{C}$ ). The stability is a result of the low aromatic stabilization energy of these compounds. The photostability was also checked for the compounds $\mathbf{1}$ and $\mathbf{2}$ in solution [9]. The compound $\mathbf{1}$ was found to be extremely photostable in methylcyclohexane and the number of repeatable cycles was $>10^{4}$. The compound 2 is characterized by a signific antly smaller number of cycles -200 in hexane in air, and it increased up to 850 under vacuum [9]. Doping different polymer matrices with photochromic molecules is certainly a way to fabricate compact and cheap materials for applications, but the properties of the obtained composites may be very different with respect to the very good fatigue resistance of compounds $\mathbf{1}$ and $\mathbf{2}$ in liquid phase. The changes of the properties could be caused by e. g. interactions between photochromic molecules and hosting matrix or/and chemical

${ }^{1}$ Italian Institute of Technology, Nanophysics Department, Genova, Italy

${ }^{2}$ Center for Biomolecular Nanotechnology - Italian Institute of Technology, Lecce, Italy

*Contacting author: Italian Institute of Technology, Nanophysics Department, Genova, Italy, phone: +39 01071781 765; fax: +39 010720 321; e-mail: elena.samoylova@, iit. it reactions in the polymer or in the molecule. In this work we investigated fatigue resistance (photostability) of composites consisting of two different polymers, PEMMA (poly(ethyl methacrylate-co-methyl acrylate) and PMMA (polymethyl methacrylate) doped with two diarylethene molecules (fig.1). The polymers have similar structure but quite different glass transition temperature $\left(\mathrm{T}_{\mathrm{g}}\right)$ which could possibly affect the photoconversion between the two photochromic forms inside the matrix. The photostability was estimated by number of repeatable coloration/decoloration cycles. The efficiency of the photoconversion was controlled by absorption spectroscopy after every 5 or 10 cycles of the UV-VIS laser irradiation. The irradiation of the samples was carried out in normal atmosphere and under reduced-oxygen condition to suppress formation of photooxidation products. We demonstrate that the hosting matrix affects the performance of a photoswitch, under both normal and reduced-oxygen conditions. Alternative reasons for the reduced number of cycles are also discussed.

\section{Matherials ANd Methods}

The samples were prepared by mixing powders of polymers (PMMA - Sigma Aldrich, MW 120000, PEMMA (PARALOID $^{\mathrm{TM}}$ B-72) - Rohmihaas) and photochromic molecules (1A - 1,2-Bis[2-methylbenzo[b]thyophen-3-yl]3,3,4,4,5,5-hexafluoro-1-cyclopentene, $\quad \mathbf{2 A}$ - 1,2-Bis[2,4dimethyl-5-phenyl-3-thienyl]-3,3,4,4,5,5-hexafluoro-1-

cyclopentene, TCI, $>97 \%$ purity) in chloroform. The amount of photochromic molecules in polymers was kept at $10 \% \mathrm{wt}$. The films were prepared by spin coating the solution on $1 \mathrm{~mm}$ thick quartz substrates and the film thickness was in the range of 1.5-

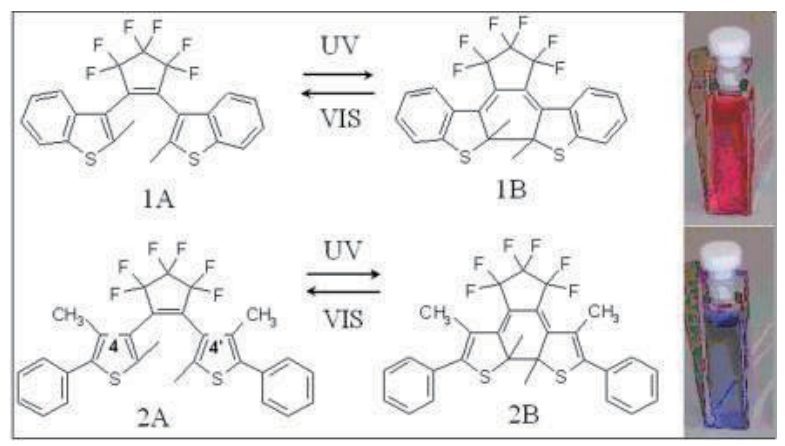

Fig.1. Chemical structure of the two photochromic compounds used in this work: the open colorless forms $1 \mathrm{~A}$ and $2 \mathrm{~A}$ are converted into closed colored $1 \mathrm{~B}$ and $2 \mathrm{~B}$ forms by UV light, and the reversed reaction is initiated by visible light. 
$1.7 \mu \mathrm{m}$ as determined with a profilometer. For the irradiation, two CW laser sources were used: the UV was delivered by the He:Cd laser $(\lambda=325 \mathrm{~nm})$ and the green light was generated by a diode laser $(\lambda=561 \mathrm{~nm})$. The irradiation wavelengths were chosen to match the absorption bands of the open and closed forms of both photochromic molecules. The photoconversion between the two forms was controlled using absorption spectroscopy. The absorption of photochromic films was checked with a spectrophotometer (Varian, Cary 6000i). To obtain fatigue resistance curves as a function of the corresponding number of cycles, we extracted the value of the peak of the absorbance band in the visible range of the UVirradiated samples, after subtraction of the absorption contribution at the same wavelength of the non-irradiated sample. All values were normalized over the one obtained after the first irradiation. The final cycle at which a composite was considered to be still working was assumed to be that in which the signal of those plots dropped to $80 \%$ of the maximum signal.

\section{RESULTS AND DISCUSSION}

To observe a photoconversion between the two isomeric forms of the photochromic molecules, their absorption spectra in polymer matrix before and after irradiation were recorded. Absorption spectra of the PMMA polymer films doped with a molecule $\mathbf{1}$ and $\mathbf{2}$ in the open and closed forms are shown in fig.2. The open-ring conformers (colorless form) are characterized by strong absorption in the UV spectral range (< $350 \mathrm{~nm})$. Irradiation with a UV laser induces formation of lower-lying excited states upon a conformational change and results in additional absorption bands observed in the visible spectral range and centered at $527 \mathrm{~nm}$ for the compound $\mathbf{1 B}$ and at $570 \mathrm{~nm}$ for the compound $\mathbf{2 B}$.

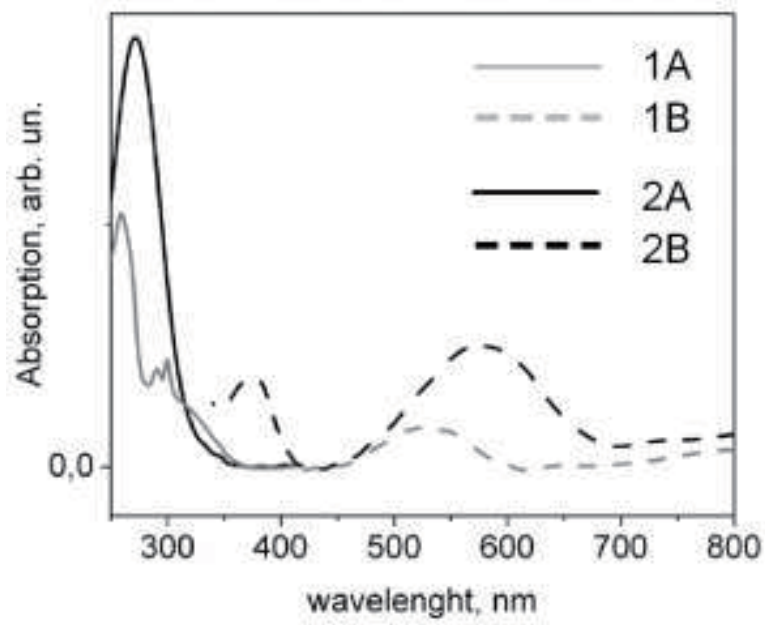

Fig. L. Aosorpuon specira or tne open (sona mnes) and ciosed (aasned lines) forms of compound $\mathbf{1}$ (gray) and $\mathbf{2}$ (black) in PMMA matrix. The spectral intensities of the closed forms are multiplied by a factor of 2 for better visibility and correspond to the maximum UV-irradiation time of the films.
The formation of a closed-ring conformer causes coloration of the samples: reddish - for $\mathbf{1}$ and indigo - for $\mathbf{2}$ (shown for solutions in fig.1). Fig.3 shows how the spectral intensities of the open and closed forms change as a function of irradiation time. In this figure, only an example of compound 2 in PMMA is shown, but identical measurements were carried out for all investigated samples. The UV-irradiation time was varied in steps of $20 \mathrm{sec}$ until the saturation of the absorption signal at $570 \mathrm{~nm}$ was reached at $80 \mathrm{sec}$. In the subsequent experiments, in each cycle the irradiation time used was the one leading to the rise of the absorption band up to the $90 \%$ of its saturation value. The reversed ring-opening reaction was initiated by irradiating the same area of the sample with the green light until the films became colorless again.

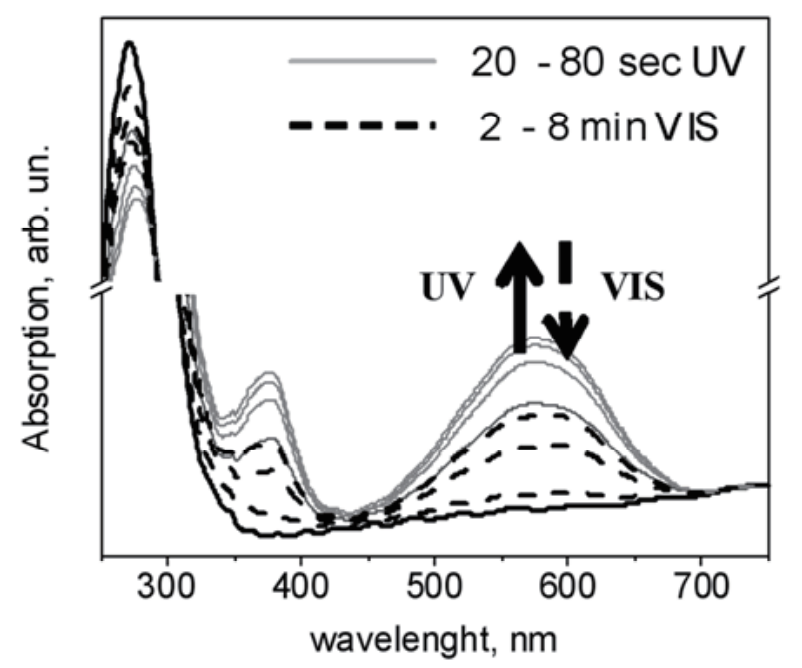

Fig. 3. Absorption spectra of the open (black thick line) and closed forms (gray and dashed lines) of compound 2 in PMMA matrix. Intensity of two new peaks increases upon UV irradiation indicating the ring closing (gray lines). The ring opens again upon irradiation with a green laser (dashed lines).

The irradiation time for one coloration and decoloration cycle was measured for each sample separately. It was noticed that the time necessary for ring closure (coloration) varied only between 20 and $50 \mathrm{sec}$ for both compounds in both different matrices. Much bigger variation in the VIS-irradiation time was observed for the ring opening reaction (decoloration) of the two molecules. This time fluctuated between $30 \mathrm{sec}$ and 8 min depending on the polymer matrix. For both UV and VIS irradiation, the samples doped in the harder PMMA polymer required longer irradiation times for photo-transformations.

The measurement results of coloration-decoloration cycles are presented in fig. 4. The experiment was carried out in normal atmosphere and under reduced-oxygen condition by blowing $\mathrm{N}_{2}$ gas on the samples surface upon laser irradiation. In fig. $4 \mathrm{a}$ the fatigue resistance curves for the compound 1 in PEMMA and PMMA polymers are plotted. A significantly different behavior in two polymers was observed. In the absence of the 
nitrogen flow the final number of cycles in both polymers remained similar, about 80 . When the gas flow was applied, the softer polymeric PEMMA matrix showed an increase in the number of coloration-decoloration cycles of the compound $\mathbf{1}$. With a linear approximation, the final number of cycles was estimated to be 300 . On the contrary, no significant difference in the number of cycles (80) was observed in PMMA which is characterized by a higher $T_{g}$. Fatigue res istance curves of the photochromic compound 2 in PEMMA and PMMA polymers with and without $\mathrm{N}_{2}$ flow are plotted in fig. $4 \mathrm{~b}$. The number of coloration-decoloration cycles was very low in the PMMA matrix and did not exceed 5 cycles. Oxygen removal in this case slightly increased the number of cycles to 9. In the PEMMA polymer we obtained a higher number of cycles -19 , and it remained unchanged in air and under a reduced-oxygen condition. Table 1 summarizes the obtained number of coloration-decoloration cycles for all experimental conditions.

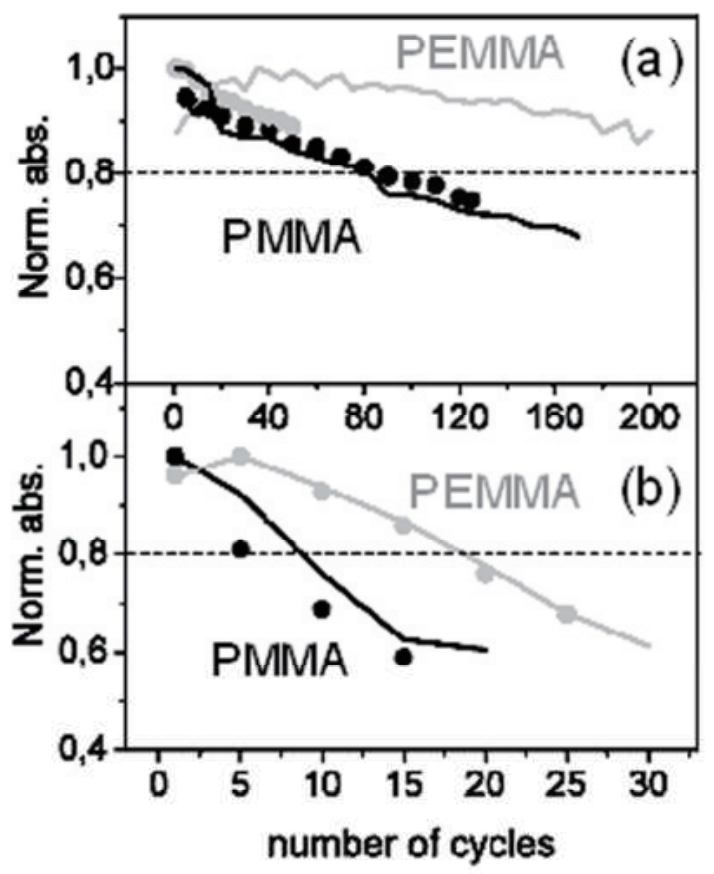

Fig. 4. Fatigue resistance curves of compounds 1 (a) and 2 (b) in PEMMA (gray) and PMMA (black) polymers in absence (circles) and presence (lines) of nitrogen flow.

To explain the observed fatigue resistances of the two compounds studied in the two different polymers, we have to consider several physical and chemical factors.

Hosting matrix properties. Despite very similar molecular structure, the two polymers have significantly different glass transition temperatures: $36{ }^{\circ} \mathrm{C}$ for PEMMA and $107{ }^{\circ} \mathrm{C}$ for PMMA [9]. During film preparation, when a polymer undergoes a transition from a liquid to an amorphous solid state, formation of free-volume structures (pores, cavities, channels) takes place [13]. The cavity size decreases with increasing $T_{g}$. And therefore in our case, one should expect smaller cavities for the PMMA polymer. Among the two, PMMA is the much more investigated material. According to Patricío et al. [14], the average radius of cavities, as measured with positron annihilation lifetime spectroscopy is $0.306 \mathrm{~nm}$ for PMMA, corresponding to a volume of $0.120 \mathrm{~nm}^{3}$. For the PEMMA polymer such measurements were not found in literature. The lower $\mathrm{T}_{\mathrm{g}}$ allows us to suggest however that larger cavities should be formed in PEMMA. Moreover, there is another parameter influencing the cavity size that should be taken into account - the polymer chain length. Longer chain polymers are characterized by smaller cavities. Unfortunately, this parameter is only known for the PMMA (MW 120000) in this work. Taking into account different cavity sizes in two polymeric matrices, one might assume different hosting ways for the molecules $\mathbf{1}$ and $\mathbf{2}$. The structure of the compound $\mathbf{2}$ was reported by Irie et al. [15]. It has an elongated shape, with the longer axis that measures $1.39 \mathrm{~nm}$. The compound 1 should be slightly smaller, as we can deduce by its chemical structure. This means that both molecules are somewhat larger than the cavities in PMMA. That would imply that most likely the major part of photochromic molecules is incorporated into the polymer between the polymeric chains and not in the free cavities. Since a photochromic reaction requires a certain space to occur, due to the structural deformation of the molecule a partial damage can occur during a photo-conformation upon UV irradiation, caused by the interaction with the surrounding polymer. Additionally, an important role plays the polymer mobility which is higher for polymers with lower $\mathrm{T}_{\mathrm{g}}$. In our experiment, PEMMA polymer has a $T_{g}$ value which is very close to the room temperature and therefore is more mobile than the PMMA polymer. This might result in a better accommodation of photochromic molecules in PEMMA matrix and less damage occurring during the photoreaction.

Temperature effect. All experiments described here were done under normal conditions, with the temperature variation of about $\pm 1{ }^{\circ} \mathrm{C}$. Irradiation of the samples with the $\mathrm{CW}$ lasers might increase slightly the temperature of the films. On the other hand, nitrogen flow cools the samples. Therefore, one can consider two effects: enlargement of the cavities (due to increased polymer mobility) and "freezing" the cavities. The first effect due to heating should be dominant in the beginning of cycled irradiation, whereas the second effect influences the thermalization temperature of the composites after several irradiations. Since the $T_{g}$ of PEMMA is very close to the room temperature, this joint temperature effect should be much easier to observe in this polymer. We therefore assume that the shape of the curve in fig. 4a (gray line) could be explained by heating-cooling effect. In the beginning of the curve one can observe a rise, and then after approx. 40 cycles the curve decays slowly. This indicates that within first 40 irradiation cycles the absorption intensity of the closed form increases, possibly due to improved accommodation of the molecules 
inside the polymer. However, an exact temperature measurement on the irradiation area is quite difficult under our experiment conditions. In the PMMA polymer the $T_{g}$ is much higher. The mentioned above heating-cooling effect leads to an equilibrium temperature which remains well above the $T_{g}$ during the experiment, also meaning a low mobility of the polymer. Mechanical restriction during photoconversion could be the major reason for molecular damage and therefore low number of cycles in PMMA (s ee fig.4, black lines and circles). Chemical reactivity. This factor can be divided into two contributions. The first one is due to chemical reactivity of the photochromic molecules and the consequent formation of photoproducts due to a rearrangement of chemical bonds during the photochromic reaction driven by UV light. Upon this rearrangement undesirable photoinduced side reactions can take place. As mentioned in [10], a compound similar to compound 2 of this work but lacking the methyl groups in 4and 4'-positions can undergo an efficient reaction of endoperoxide formation (chemical species containing -O-O group inside a bigger molecule) in presence of oxygen. As a result of this reaction, two thiophene rings merge together to form two six-membered rings. As suggested in [10], formation of these rings can also accelerate the photodamage process. It was also noted that methyl groups in the 4- and 4'- positions of the thiophene rings are considered to reduce or even prevent formation of six-membered rings. This photooxidation process was described however in hexane solution. On the contrary, for the crystalline phase of the compound 2 lacking the methyl groups, the photochromism is preserved and formation of the photoproduct was not observed even after 10000 cycles [10]. Possibly, due to limited degrees of freedom of the molecule, rearrangement of the thiophene rings is prohibited. In a polymer matrix (this work), we might have intermediate behavior of the compound $\mathbf{2}$ between a liquid phase and a crystalline phase, and oxidation photoproducts could be formed, thus reducing the number of coloration-decoloration cycles. The second contribution could come from possible interactions between the photochromic molecules and the polymeric matrix. As it was shown by Lin [9], PMMA polymer doped with spiropyran can have dipole-dipole interaction due to high polarity of PMMA polymer which influences the photochromic reaction. If such interaction between diarylethene compounds and polymer matrices takes place, the forward or backward photochromic reactions could be slowed down [9]. Possibly this could explain the longer VIS-irradiation time of PMMAphotochromic films.

To summarize, we suggest that all mentioned causes - matrix properties, temperature change, oxidation damage and matrixmolecule interaction could influence the fatigue resistance properties of photochromic material investigated here. However, the best result reported here is obtained for PEMMA polymer doped with the compound 1. From this we can conclude that to get a photochromic material with best fatigue resistance, the following conditions should be fulfilled: (1) the polymer should have a low $\mathrm{T}_{\mathrm{g}}$, (2) photooxidation damage should be minimized by removing oxygen or choosing an extremely photochemically stable compound (for compound $\mathbf{1}$ intensive formation of photoproducts was not reported), (3) polymer matrix and photochromic molecules should interact as less as possible. Depending on desired application, the property of fatigue resistance should be checked before fabricating a device to find out the best working conditions, also taking into account other parameters not considered in the present work, like e.g. optimal photochromic molecule concentration in the host matrix. The presented results are a part of an on-going work on optical memories, and other characteristics relevant for the project are now under investigation.

Tab.1. Number of coloration-decoloration cycles for compounds $\mathbf{1}$ and $\mathbf{2}$ in PEMMA and PMMA polymers as estimated from the measurements described in the text.

\begin{tabular}{lcc}
\hline \multicolumn{1}{c}{ Material } & $\begin{array}{c}\text { Number of } \\
\text { cycles }\left(\mathbf{n o} \mathbf{~ N}_{\mathbf{2}}\right)\end{array}$ & $\begin{array}{c}\text { Number of cycles } \\
\left.\mathbf{( N}_{\mathbf{2}}\right)\end{array}$ \\
\hline $\mathbf{1}$ in PEMMA & $\sim 80$ & $>300$ \\
\hline $\mathbf{1}$ in PMMA & 80 & 80 \\
\hline $\mathbf{2}$ in PEMMA & 19 & 19 \\
\hline $\mathbf{2}$ in PMMA & 5 & 9 \\
\hline
\end{tabular}

\section{REFERENCES}

[1] Y. Chen, T. Li, M. Fan, X. Mai, H. Zhao, D. Xu, "Photochromic filgides for multi-level recording", Mat. Sci. \& Eng. B, 123, pp.53-56, 2005.

[2] Y. Liang, A. S. Dvornikov, P. M. Rentzepis, "Solvent and ring substitution effect on the photochromic behaviour of 2-indolylfulgide derivatives", J. Photochem. Photobiol. A: Chemistry, 125, pp.79-84, 1999.

[3] T. Cordes, S. Malkmus, J. A. Digirolamo, W. J. Lees, A. Nenov, R. Vivie-Riedle, M. Braun, W. Zinth, „Acceleration and efficient photochemistry from higher excited electronic states in fulgide molecules“, J. Phys. Chem. A, 112 (51), pp. 13364-13371, 2008.

[4] S. Malkmus, F. O. Koller, B. Heinz, W. J. Schreier, T. E. Schrader, W. Zinth, C. Schulz, S. Dietrich, K. Rück-Braun, M. Braun, „Ultrafast ring opening reaction of a photochromic indolyl-fulgimide“, Chem. Phys. Lett, 417, pp. 266-271, 2006.

[5] M. Momasulo, S. Sortino, F. M. Raymo, "A new family of photochromic compounds based on the photoinduced opening and thermal closing of [1,3] oxazine rings", J. Photochem. Photobiol. A: Chemistry, 200, pp.4449, 2008.

[6] G. Berkovic, V. Krongauz, V. Weiss, "Spiropyrans and spirooxazines for memories and switches", Chem. Rev. 100 (5), pp. 1741-1754, 2000.

[7] A. Athanassiou, M. Kalyva, K. Lakiotaki, S. Georgiou, C. Fotacis, "Alloptical reversible actuation of photochromic-polymer microsystems", Adv. Mater. 17, No. 8, pp. 988-992, 2005.

[8] A. Athanassiou, K. Lakiotaki, V. Tornari, S. Georgiou, C. Fotacis, "Photocontrolled mechanical phenomena in photochromic doped polymeric systems", Appl.Phys. A. 17, pp. 97-100, 2003.

[9] J.-S. Lin, "Interaction between dispersed photochromic compound and polymer matrix" Eur. Polym.J, 39 (8), pp. 1693-1700, 2003.

[10] M. Irie, "Diarylethenes for Memories and Switches," Chem. Rev. Vol. 100, pp. 1685-1716, 2000.

[11] S. Shim, I. Eom, T. Joo, E. Kim, K. S. Kim, "Ring closure reaction dynamics of diarylethene derivatives in solution" J. Phys. Chem. A. 111(37), pp. 8910-8917, 2007.

[12] M. Irie, T. Lifka, S. Kobatake, N. Kato, "Photochromism of 1,2-Bis(2methyl-5-phenyl-3-thienyl)perfluorocyclopentene in a single crystalline phase" J. Am. Chem. Soc. 122, pp. 4871-4876, 2000. 
[13] J. Bohlen, J. Wolff, R. Kirchheim, "Determination of free-volume and hole number density in polycarbonates by positron lifetime spectroscopy" Macromolecules 32, pp. 3766-3773, 1999.

[14] P. S. O. Patrício, G. G. Silva, J. C. Machado "Free volume properties of thermoplastic polyurethane/polymethylmethacrylate blends: evidence of interchain interaction" J. Appl. Polym. Sci. 105, pp. 641-646, 2007.

[15] M. Irie, S. Kobatake, M. Horichi, "Reversible surface morphology changes of a photochromic diarylethene single crystal by photoirradiation"Science 291, pp. 1769-1772, 2001. 